Published in final edited form as:

Chem Mater. 2005 August 9; 17(16): 4256-4261.

\title{
Sulfide-Arrested Growth of Gold Nanorods
}

\author{
Daniel A. Zweifel and Alexander Wei ${ }^{*}$ \\ Department of Chemistry and the Birck Nanotechnology Center, Purdue UniVersity, 560 Oval Drive, \\ West Lafayette, Indiana 47907-2084
}

\begin{abstract}
The growth of gold nanorods can be arrested at intermediate stages by treatment with $\mathrm{Na}_{2} \mathrm{~S}$, providing greater control over their optical resonances. Nanorods prepared by the seeded reduction of $\mathrm{AuCl}_{4}$ in aqueous cetyltrimethylammonium bromide solutions in the presence of $\mathrm{AgNO}_{3}$ typically exhibit a gradual blueshift in longitudinal plasmon resonance, over a period of hours to days. This "optical drift" can be greatly reduced by adding millimolar concentrations of $\mathrm{Na}_{2} \mathrm{~S}$ to quench nanorod growth, with an optimized sulfur:metal ratio of 4:1. The sulfide-treated nanorods also experience a marked redshift as a function of $\mathrm{Na}_{2} \mathrm{~S}$ concentration to produce stable plasmon resonances well into the nearinfrared. Sulfide treatment permitted a time-resolved analysis of nanorod growth by transmission electron microscopy, revealing two distinct periods: an initial growth burst $(t<15 \mathrm{~min})$ that generates dumbbell-shaped nanorods with flared ends and a slower phase $(t>30 \mathrm{~min})$ favoring growth around the midsection, leading to nanorods with the more familiar oblate geometry. The blueshift in plasmon resonance that accompanies the dumbbell-to-oblate shape transition correlates more strongly with changes in the length-to-midsection $\left(L / D_{1}\right)$ ratio rather than the length-to-end width $\left(L / D_{2}\right)$ ratio, based on the empirical relationship introduced by El-Sayed and co-workers.
\end{abstract}

\section{Introduction}

Gold nanostructures offer excellent prospects for probing biological systems. The base material is biochemically inert and can be manipulated at nanometer length scales to support localized surface plasmon resonances at visible and near-infrared (NIR) wavelengths. The latter is wellknown to penetrate biological tissues with relatively high transmittivities: wavelengths shorter than $800 \mathrm{~nm}$ are absorbed by hemoglobin and melanin, and wavelengths greater than $1300 \mathrm{~nm}$ are strongly attenuated by water. Plasmonic nanomaterials with NIR resonances are currently being investigated as biomolecular sensors, ${ }^{1-3}$ as contrast agents for biomedical imaging, ${ }^{4}$ and as mediators of photodynamic therapy. ${ }^{5}$

Gold nanorods are particularly well-suited for biosensing and biomedical imaging at NIR frequencies because their plasmon resonance modes can be tuned as a function of aspect ratio. 6-9 Nanorods have been synthesized in the presence of micelle-forming surfactants such as cetyltrimethylammonium bromide (CTAB) by electrolysis at cathodic potentials ${ }^{10}$ or by the chemical reduction of $\mathrm{AuCl}_{4}$ ions. ${ }^{11-15}$ With regard to the latter, several groups have shown that seeded growth under mildly reducing conditions can produce nanorods with exquisite control over size and shape, as demonstrated by transmission electron microscopy (TEM). 11-13 Sau and Murphy have recently demonstrated that nanorods with aspect ratios of 2-4 can be formed in nearly quantitative yields when $\mathrm{AgNO}_{3}$ is used as an additive; in some cases, a dumbbell-like morphology appears to be favored over the cylindrically symmetric oblate geometry. ${ }^{11 \mathrm{c}}$ Dumbbell-shaped nanostructures have also been reported by Liu and

\footnotetext{
*Corresponding author e-mail: alexwei@purdue.edu.

Supporting Information Available: Additional TEM images of Au nanorods before and after treatment with $0.2 \mathrm{mM} \mathrm{Na} 2 \mathrm{~S}$. This material is available free of charge via the Internet at http://pubs.acs.org.
} 
GuyotSionnest 16 and by Chang and co-workers, ${ }^{17}$ who used preformed gold nanorods to nucleate the reduction of silver ions. In contrast, a study by Mulvaney and co-workers did not reveal any dumbbell-shaped particles when nanorods were prepared in the absence of $\mathrm{AgNO}_{3} \cdot{ }^{13}$

The seeded growth of $\mathrm{Au}$ nanorods in the presence of $\mathrm{AgNO}_{3}$ promises to be a practical synthesis with respect to both yield and uniformity. However, we have observed that the plasmon resonances of such nanorods are often unstable and can drift toward shorter wavelengths by as much as $100 \mathrm{~nm}$ over a period of hours to days. Applications involving plasmon-enhanced spectroscopies require substrates with stable, well-defined resonances and line shapes: a shift of a few nanometers can reduce signal intensities by orders of magnitude, particularly if nonlinear optical enhancements are involved. $9,18,19$ The nanorods' optical instability thus becomes an important issue to address, as it limits them from reaching their full technological potential.

Here we demonstrate that the optical drift in Au nanorods can be greatly reduced by the controlled addition of $\mathrm{Na}_{2} \mathrm{~S}$. Sulfide ions have a strong affinity for heavy metals in their ionic and metallic states and are commonly used in ore beneficiation and metal surface passivation. 20 The sulfide treatment provides an additional benefit by modulating the dielectric function at the nanorod surface, shifting the plasmon resonances toward longer wavelengths. The sulfide-treated nanorods are stable at room temperature after isolation from the reaction mixture and can be stored for months without degradation. Finally, the sulfide treatment permits us to obtain reliable insights on nanorod growth after nucleation, enabling a correlation between nanostructure and optical resonance.

\section{Experimental Section}

\section{Reagents}

Cetyltrimethylammonium bromide (CTAB), $\mathrm{AgNO}_{3}$, and $\mathrm{HAuCl}_{4}$ were purchased from Alfa Aesar and used as supplied. The quality of the CTAB is particularly important as minor impurities (most likely cetyldimethylamine) can result in much faster rates of reduction, with subsequently poor control over the reaction kinetics. Deionized water was obtained using an ultrafiltration system (Milli-Q, Millipore) with a measured resistivity above $18 \mathrm{M} \Omega \cdot \mathrm{cm}$ and passed through a $0.22-\mu \mathrm{m}$ filter to remove particulate matter.

\section{Au Nanorod Synthesis}

Growth and seed solutions were prepared according to the protocol described by Nikoobakht and El-Sayed. ${ }^{12}$ Au seeds $\left(d_{\mathrm{av}}=3.5 \mathrm{~nm}\right)$ were prepared by mixing equal portions of an aqueous $0.2 \mathrm{M} \mathrm{CTAB}$ solution $(5 \mathrm{~mL})$ and a $0.5 \mathrm{mM} \mathrm{HAuCl}_{4}$ solution $(5 \mathrm{~mL})$ at room temperature in a clean glass vial, followed by an injection of a cold $\left(0{ }^{\circ} \mathrm{C}\right) 10 \mathrm{mM} \mathrm{NaBH}_{4}$ solution $(0.6 \mathrm{~mL})$. The reaction mixture was stirred for 2 min with a magnetic stir bar prior to its addition to the growth solution.

The nanorod growth solution was prepared by combining an aqueous solution of $0.2 \mathrm{M} \mathrm{CTAB}$ $(5 \mathrm{~mL})$ with $4 \mathrm{mM} \mathrm{AgNO} 3(0.24 \mathrm{~mL})$ in a $40-\mathrm{mL}$ vial, followed by immersion in an ultrasonic cleaning bath (Branson, $42 \mathrm{kHz}$ ) for $10 \mathrm{~min}$. The growth solution was then treated with $1 \mathrm{mM}$ $\mathrm{HAuCl}_{4}(5 \mathrm{~mL})$ and $78.8 \mathrm{mM}$ ascorbic acid $(70 \mu \mathrm{L})$, followed by an injection of the seed solution ( $12 \mu \mathrm{L}$; see above). The reaction mixture was stirred by hand for $30 \mathrm{~s}$ and then allowed to sit at room temperature for a designated period of time $(t)$ before being quenched with 15 $\mathrm{mL}$ of $\mathrm{Na}_{2} \mathrm{~S}$ solution at various concentrations, with the mole ratio of $\mathrm{S}$ to metal ( $\mathrm{Au}$ and $\mathrm{Ag}$ ) ranging from 1:2 $(0.125 \mathrm{mM})$ to $4: 1(1 \mathrm{mM})$. The sulfide-treated nanorods were transferred to plastic centrifuge tubes and subjected to centrifugation at $12500 \mathrm{~g}$ ( $1 \mathrm{~min}$ at maximum speed), then decanted from the supernatant and redispersed in deionized water $(6-8 \mathrm{~mL})$, and 
characterized by optical absorption spectroscopy using a Cary-50 visible-NIR spectrophotometer (Varian) with a 1-cm cell path length.

\section{Results and Discussion}

In the course of a typical nanorod synthesis, plasmon resonance peaks appeared within 15-20 $\mathrm{min}$ in the NIR and reached their maximum absorbance intensities after 50-60 min accompanied by a blueshift of the longitudinal plasmon peak toward the visible, in accord with the recent report of Sau and Murphy (see Figure 1a). ${ }^{11 \mathrm{c}}$ It must be mentioned that the resonance wavelengths produced after a given time interval varies from batch to batch and appear to be quite sensitive to nucleation conditions. We have observed that nanorod batches prepared and isolated under apparently identical conditions can differ in their optical resonances by $5-30$ $\mathrm{nm}$, precluding an exact comparative analysis for different reaction conditions. Nonetheless, the same trends are always observed with respect to the blueshift during the course of the reaction, as well as the subsequent optical drift over time. Therefore, all discussions concerning the optical stability of nanorods are based on post-synthetic changes in plasmon resonance, relative to aliquots of freshly prepared, untreated nanorods.

Our initial attempts to isolate nanorods with specific optical resonances involved high-speed centrifugation, followed by redispersion of the precipitants in deionized water. ${ }^{12}$ This proved not to be effective: the optical absorption of the recovered nanorods continued to drift toward shorter wavelengths over a period of several days, even after two consecutive cycles of centrifugation/redispersion (C/R; see Figure 1b). TEM analysis further revealed that the conditions and timing of sample preparation had a strong influence over nanorod size and shape: particles cast directly from the seeded solutions onto TEM substrates (sample A) exhibited dumbbell-like morphologies, whereas those collected after one $\mathrm{C} / \mathrm{R}$ cycle (samples $\mathrm{B}$ and $\mathrm{C}$ ) underwent a gradual structural transition to a cylindrically symmetric form (see Figure 2). A likely explanation for this evolution is that the unreacted Au ions were not freely suspended in solution but were adsorbed instead onto the surfaces of CTAB micelles, which co-precipitated with the nanorods upon centrifugation and could be subsequently redispersed. The virtual absence of free Au ions in the presence of CTAB and the very low solubility of the $\mathrm{Au}-\mathrm{CTAB}$ complex have been confirmed in previous studies. ${ }^{21}$ Mulvaney and co-workers have recently provided strong evidence for a nanorod elongation mechanism that involves a collision-activated process with Aumicelle complexes. ${ }^{13}$

Particle size analysis of samples A-C provided some additional insights in the changes of nanorod structure as a function of sample preparation (see Table 1). Two trends are immediately apparent: (i) the nanorod lengths $(L)$ and end widths $\left(D_{2}\right)$ decrease from samples A-C, and (ii) the midsection-to-end width ratios $\left(D_{1} / D_{2}\right)$ increase from A-C. The first trend indicates a decrease in residual metal ion, which contribute to nanorod growth even while drying on the TEM substrates, whereas the second trend suggests a late growth stage around the nanorod midsection. The $D_{1} / D_{2}$ ratios may be overestimated by an acceleration in lateral $\left(D_{1}\right)$ growth during TEM sample preparation: as the wetting layer evaporates, dewetting forces can concentrate residual solute into regions of negative curvature, promoting localized reduction of $\mathrm{Au}(\mathrm{I})$ ions. This lack of synthetic control underscores the difficulty of characterizing the nanorods in the presence of unreacted Au ions.

Treating the reaction mixtures with sodium sulfide was found to be an effective means of quenching nanorod growth. Reactions were set up in parallel and treated with $\mathrm{Na}_{2} \mathrm{~S}$ solutions at $t=15,30$, and $50 \mathrm{~min}$ after addition of the seed solution, followed 15 min later by centrifugation and redispersion of the nanorods in deionized water. The mole ratio of $\mathrm{S}$ to the total metal content ( $\mathrm{Au}$ and $\mathrm{Ag}$, or M) was varied from 1:2 to 4:1. As before, a blueshift in 
$\lambda_{\max }$ was observed during the course of nanorod growth, but this was arrested immediately upon addition of $\mathrm{Na}_{2} \mathrm{~S}$.

Monitoring the sulfide-treated nanorods over a 6-day period revealed large improvements in optical stability as a function of $\mathrm{Na}_{2} \mathrm{~S}$ addition, the effect being most pronounced for nanorods treated at an early growth stage (see Figure 3). Minimal drift was observed at a S:M ratio of $4: 1$. For nanorods quenched at $t=15 \mathrm{~min}$, the optical drift over a 6-day period was reduced from $-100 \mathrm{~nm}$ (no sulfide treatment) to $-5 \mathrm{~nm}$; for nanorods quenched at $t=50 \mathrm{~min}$, the optical drift could be completely arrested (see Table 2). Further increases in sulfide concentration did not have any benefits; in fact, excessive sulfide led to the slow disintegration of the nanorods. The reduction in optical drift with $\mathrm{Na}_{2} \mathrm{~S}$ concentration suggests that sulfide serves primarily as a scavenger for $\mathrm{Au}$ and $\mathrm{Ag}$ ions, effectively removing them from the reaction mixture. Zhang and co-workers have provided evidence that $\mathrm{AuCl}_{4}$ ions are reduced by $\mathrm{Na}_{2} \mathrm{~S}$ to form metallic Au nanoparticles rather than $\mathrm{Au}_{2} \mathrm{~S} .22$

Treating the $\mathrm{Au}$ nanorods with $\mathrm{Na}_{2} \mathrm{~S}$ also induced a concentration-dependent increase in plasmon resonance toward longer wavelengths. This redshift, which we ascribe to modulations in the nanorods' dielectric function by sulfide adsorption, is again most pronounced for nanorods quenched at an early stage of growth (see Table 2). In contrast to the slow optical drift described previously, the sulfide-induced redshift is essentially a singular event. To confirm the influence of sulfide adsorption on plasmon resonance, previously untreated nanorod dispersions (aged for several days) were treated with $\mathrm{Na}_{2} \mathrm{~S}$ and examined by absorption spectroscopy. A concentration-dependent effect on the longitudinal plasmon resonance was again observed, with redshifts up to $29 \mathrm{~nm}$ for a S:M ratio of 4:1 (see Figure 4). It is interesting to note that a redshift was not induced at the lowest $S: M$ ratio (1:2), implying that the sulfide did not reach the nanorod surface. Changes in plasmon resonance as a function of the medium dielectric are well-established for Au nanospheres ${ }^{23}$ as well as for anisotropic nanoparticles such as nanotriangles and nanorods, ${ }^{24}$ but the latter are the most sensitive to changes in their local environment. 25

TEM analysis of the sulfide-treated Au nanorod suspensions provided an unambiguous record of the time-dependent changes in nanorod morphology over the course of the reaction (see Figure 5 and Table 3; additional images in Supporting Information). Nanorods were treated at a S:M ratio of 1:2 at several different time intervals $(t=15,20,30,40$, and $50 \mathrm{~min})$, then immediately isolated and deposited onto Formvar-coated $\mathrm{Cu}$ grids. These samples differed significantly from the untreated nanorod samples prepared under similar conditions, with respect to changes in size and aspect ratios (cf. Figure 2 and Table 1).

The TEM data reveals an initial growth burst predominantly in the longitudinal direction to produce dumbbell-shaped nanorods $(t<15 \mathrm{~min})$, followed by an intermediate period of monotonic growth in both longitudinal $(L)$ and lateral $\left(D_{1}\right.$ and $\left.D_{2}\right)$ directions $(t=15-30 \mathrm{~min})$. The flaring in the dumbbell-shaped nanorods is most pronounced in the first 30 min after seed addition, with an approximately $20 \%$ difference between $D_{1}$ and $D_{2}$. A new phase appears after $t>30 \mathrm{~min}$, in which lateral growth around the nanorod midsection is faster than at the ends: while the growth rate remains unchanged in the $L$ and $D_{2}$ dimensions, growth in the $D_{1}$ dimension increases abruptly. The shift in growth kinetics to the $D_{1}$ dimension is presumably responsible for producing the final oblate geometry (cf. Figure 2c) and subsequent blueshift in plasmon resonance. Two additional observations are worth mentioning: (i) Sulfide-treated nanorods with dumbbell-like morphologies did not lose their shape over time and were thus not susceptible to CTAB-mediated reshaping. 26 (ii) Untreated nanorods isolated after $t=50$ min were not uniform in shape but observed to be a mixture of dumbbell and oblate geometries, indicating variable rates of growth in the $D_{1}$ direction (see Supporting Information). The latter 
observation suggests that nanorods with the highest uniformity can be produced by quenching their growth at an early stage, within 30 min of their nucleation.

The presence of two different lateral dimensions $D_{1}$ and $D_{2}$ raises the interesting question whether either of these would be useful for characterizing the nanorods' longitudinal plasmon resonances. Link et al. ${ }^{24}$ derived a parameterized equation based on Mie-Gans theory to establish a linear correlation between the aspect ratio of cylindrical nanorods and their plasmon resonance wavelengths:

$$
\begin{gathered}
\lambda_{\text {max }}=(33.34 R-46.31) \epsilon_{\mathrm{m}}+472.31 \\
\epsilon_{\mathrm{m}}=(103.70 R-80.20) /(33.34 R-46.31)
\end{gathered}
$$

where $R$ is the nanorod aspect ratio and $\varepsilon_{\mathrm{m}}$ is the effective dielectric medium of the surrounding micellar layer, which also varies as a function of $R \cdot{ }^{24,27}$ The application of eqs 1 and 2 is only useful to the first degree of approximation, as the nanorod shapes and micellar materials used by Link et al. ${ }^{24}$ are different from those in our study. Further modulation in the local dielectric medium by sulfide adsorption is also possible, although this effect appears to be negligible for a S:M ratio of 1:2 (cf. Table 2 and Figure 4). Nevertheless, the comparison is sufficient to demonstrate that the aspect ratio $L / D_{1}$ provides a better approximation of the longitudinal plasmon resonance wavelength than $L / D_{2}$ (see Table 4).

With respect to the growth mechanism of the dumbbell-shaped nanorods, recent evidence from the Murphy and Chang groups have shown that the $\{111\}$ facets at the nanorod ends are receptive to $\mathrm{Ag}$ ion deposition. ${ }^{11 \mathrm{c}, 17}$ In particular, the latter group has established that $\mathrm{Ag}$ metal can be deposited onto the nanorod ends by adjusting the reducing power of the growth solution. To test the role of $\mathrm{AgNO}_{3}$ in the growth of dumbbell-shaped particles, nanorods were prepared by seeded growth in the absence of $\mathrm{AgNO}_{3}$ and then quenched with $\mathrm{Na}_{2} \mathrm{~S}$ at various time intervals. The TEM images revealed several types of anisotropic nanoparticles including rods, triangles, and hexagonal and trapezoidal prisms, all of which appeared to have welldefined planar facets; however, no dumbbell-shaped particles could be observed (see Figure 6). This confirms that Ag plays a key role in dumbbell formation during the initial stages of nanorod growth. Although the precise mechanism remains to be determined, one possibility may involve the reduction of $\mathrm{Ag}$ ions by unreacted borohydride in the seed solution. ${ }^{28}$

\section{Conclusion}

The CTAB-mediated growth of Au nanorods can be arrested by adding sulfide at millimolar concentrations, resulting in chemically and optically stable species with specific NIR resonances. The stabilizing effect of sulfide can be attributed to two processes: (i) the reduction or quenching of unreacted metal salts and (ii) the adsorption of sulfide onto the nanorod surfaces. The latter has the added benefit of shifting the plasmon resonance further into the NIR spectral region. The structural and optical stability of the sulfide-treated nanorods make them attractive candidates as contrast agents for optical coherence tomography and other biophotonic applications.

\section{Supplementary Material}

Refer to Web version on PubMed Central for supplementary material.

\section{Acknowledgment}

The authors gratefully acknowledge the National Institutes of Health (EB-001777-01) and the National Science Foundation (CHE-0243496, ECS-0210445) for financial support. TEM images were taken at the Purdue Life Sciences 
Microscopy Facility. This work is in association with the Purdue Cancer Center and the Birck Nanotechnology Center at Purdue University.

\section{References}

1. Kneipp K, Haka AS, Kneipp H, Badizadegan K, Yoshizawa N, Boone C, Shafer-Peltier KE, Motz JT, Dasari RR, Feld MS. Appl. Spectrosc 2002;56:150-154.

2. (a) Sadtler B, Wei A. Chem. Commun 2002:1604-1605. (b) Zhao Y, Sadtler B, Min L, Hockerman GH, Wei A. Chem. Commun 2004;784:785.

3. Dou X, Yamaguchi Y, Yamamoto H, Doi S, Ozaki Y. J. Raman Spectrosc 1998;29:739-742.

4. Lee TM, Oldenburg AL, Sitafalwalla S, Marks DL, Luo W, Toublan FJ-J, Suslick KS, Boppart SA. Opt. Lett 2003;28:1546-1548. [PubMed: 12956374]

5. (a) Hirsch LR, Stafford RJ, Bankson JA, Sershen SR, Rivera B, Price RE, Hazle JD, Halas NJ, West JL. Proc. Natl. Acad. Sci. U.S.A 2003;100:13549-13554. [PubMed: 14597719] (b) O'Neal DP, Hirsch LR, Halas NJ, Payne JD, West JL. Cancer Lett 2004;209:171-176. [PubMed: 15159019]

6. Foss CA Jr. Hornyak GL, Stockert JA, Martin CR. J. Phys. Chem 1994;98:2963-2971.

7. van der Zande B, Böhmer MR, Fokkink LGJ, Schönenberger C. Langmuir 2000;16:451-458.

8. (a) El-Sayed MA. Acc. Chem. Res 2001;34:257-264. [PubMed: 11308299] (b) Burda C, Chen X, Narayanan R, El-Sayed MA. Chem. Rev 2005;105:1025-1102. [PubMed: 15826010]

9. Wei, A. Nanoparticles: Scaffolds and Building Blocks. Rotello, VM., editor. Kluwer Academic; New York: 2004. p. 173-200.

10. (a) Yu Y-Y, Chang S-S, Lee C-L, Wang CRC. J. Phys. Chem.B 1997;101:6661-6664. (b) Chang SS, Shih C-W, Chen C-D, Lai W-C, Wang CRC. Langmuir 1999;15:701-709.

11. (a) Jana NR, Gearheart L, Murphy CJ. J. Phys. Chem. B 2001;105:4065-4067. (b) Jana NR, Gearheart L, Murphy CJ. Adv. Mater 2001;13:1389-1393. (c) Sau TK, Murphy CJ. Langmuir 2004;20:64146420. [PubMed: 15248731]

12. Nikoobakht B, El-Sayed MA. Chem. Mater 2003;15:1957-1962.

13. Pérez-Juste J, Liz-Márzan LM, Carnie S, Chan DYC, Mulvaney P. Adv. Funct. Mater 2004;14:571579.

14. Kim F, Song JH, Yang P. J. Am. Chem. Soc 2002;124:14316-14317. [PubMed: 12452700]

15. Niidome T, Nishioka K, Kawasaki H, Yamada H. Chem. Commun 2003:2376-2377.

16. Liu M, Guyot-Sionnest P. J. Phys. Chem. B 2004;108:5882-5888.

17. Huang C-C, Yang Z, Chang H-T. Langmuir 2004;20:6089-6092. [PubMed: 15248687]

18. Genov DA, Sarychev AK, Shalaev VM, Wei A. Nano Lett 2004;4:153-158.

19. Imura K, Nagahara T, Okamoto H. J. Am. Chem. Soc 2004;126:12730-12731. [PubMed: 15469240]

20. (a) Wierse DG, Lohrengel MM, Schultze JW. J. Electroanal. Chem 1978;92:121. (b) Lezna RO, de Tacconi NR, Arvia AJ. J. Electroanal. Chem 1990;283:319.Benard, J., editor. Adsorption on Metal Surfaces. Elsevier; Amsterdam: 1983. (d) Lay MD, Varazo K, Stickney JL. Langmuir 2003;19:8416.

21. Esumi K, Torigoe K. Langmuir 1992;8:59-63.

22. Zhang JZ, Schwartzberg AM, Norman T Jr. Grant CD, Liu J, Bridges F, van Buuren T. Nano Lett 2005;5:809-810. [PubMed: 15826133]

23. Kreibig, U.; Vollmer, M. Optical Properties of Metal Clusters. Springer; New York: 1995. (b) Mulvaney P. Langmuir 1996;12:788-800.

24. Link S, Mohamed MB, El-Sayed MA. J. Phys. Chem. B 1999;103:3073-3077.

25. For a comparison between Ag nanospheres, nanotriangles, and nanorods, see:McFarland AD, van Duyne RP. Nano Lett 2003;3:1057-1062.

26. Aguirre CM, Kaspar TR, Radloff C, Halas NJ. Nano Lett 2003;3:1707-1711.

27. Revisions to eqs 1 and 2 have been suggested by Wang and co-workers, using a smaller effective value for $\varepsilon_{\mathrm{m}}$ (see:Yan B, Yang Y, Wang YJ. J. Phys. Chem. B 2003;107:9159.). However, $\lambda_{\max }$ values calculated using their revised formulation are significantly underestimated for aspect ratios greater than 3, when compared with the experimental values.

28. Mulvaney and co-workers have commented on the importance of decomposing excess borohydride to avoid inadvertent nucleation; see ref 13 . 


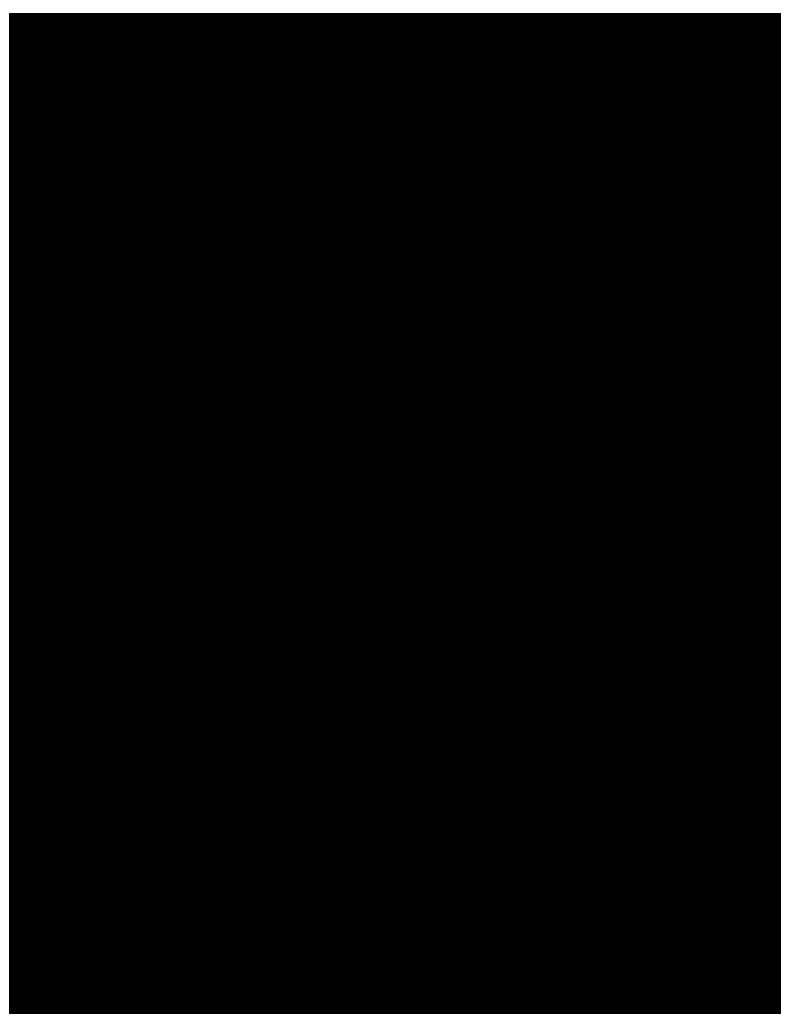

Figure 1.

Normalized absorbance spectra of Au nanorods subjected to centrifugation and redispersion $(\mathrm{C} / \mathrm{R})$ in deionized water. (a) Nanorods subjected to $\mathrm{C} / \mathrm{R}$ at various intervals after seed addition $(t=15,20,30,40$, and $50 \mathrm{~min})$. (b) Nanorods $(t=25 \mathrm{~min})$ after one or two C/R cycles, with spectra taken immediately after $\mathrm{C} / \mathrm{R}$ (fresh) and several days afterward (aged). 


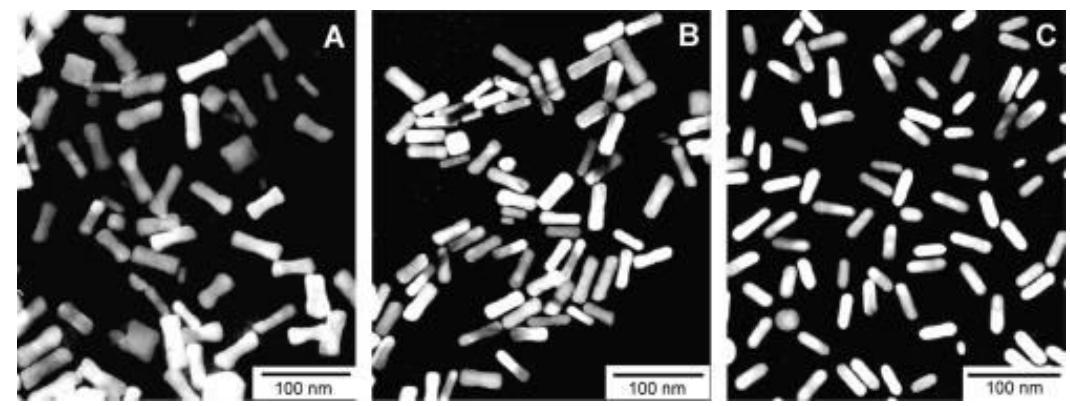

Figure 2.

TEM images (Philips CM-10, $80 \mathrm{kV}$ ) of Au nanorods isolated 30 min after seed addition. Nanorod suspensions were cast onto carbon-coated $\mathrm{Cu}$ grids, then blotted after $30 \mathrm{~s}$ and dried in air. Sample A: Nanorods deposited directly from the reaction mixture. Sample B: Nanorods subjected to $C / R$ in deionized water, just prior to sample preparation. Sample C: Same batch of nanorods as in $\mathrm{B}$, aged $24 \mathrm{~h}$ prior to sample preparation. 

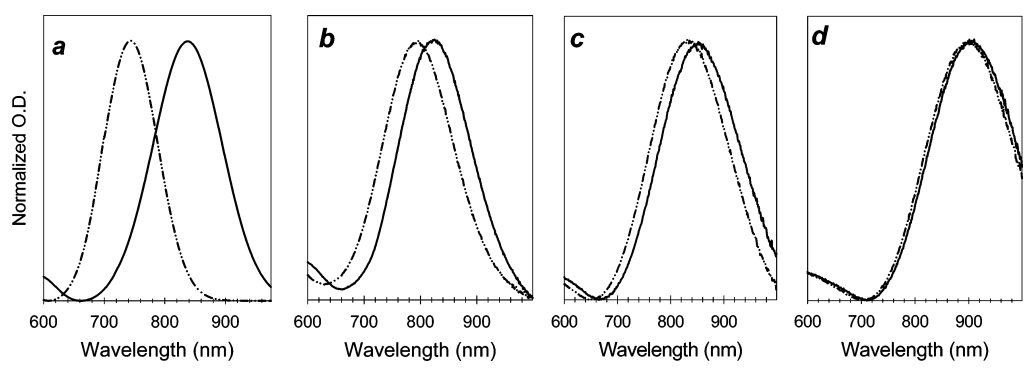

Figure 3.

Time-dependent changes in the plasmon resonance of sulfide-treated Au nanorods, quenched at $t=15 \mathrm{~min}$. Absorption spectra of nanorods were obtained immediately after one C/R cycle in deionized water (solid line) and after 6 days (dashed line). (a) no sulfide addition; (b) S:M $=1: 2 ;$ (c) $\mathrm{S}: \mathrm{M}=1: 1 ;$ (d) $\mathrm{S}: \mathrm{M}=4: 1$. 


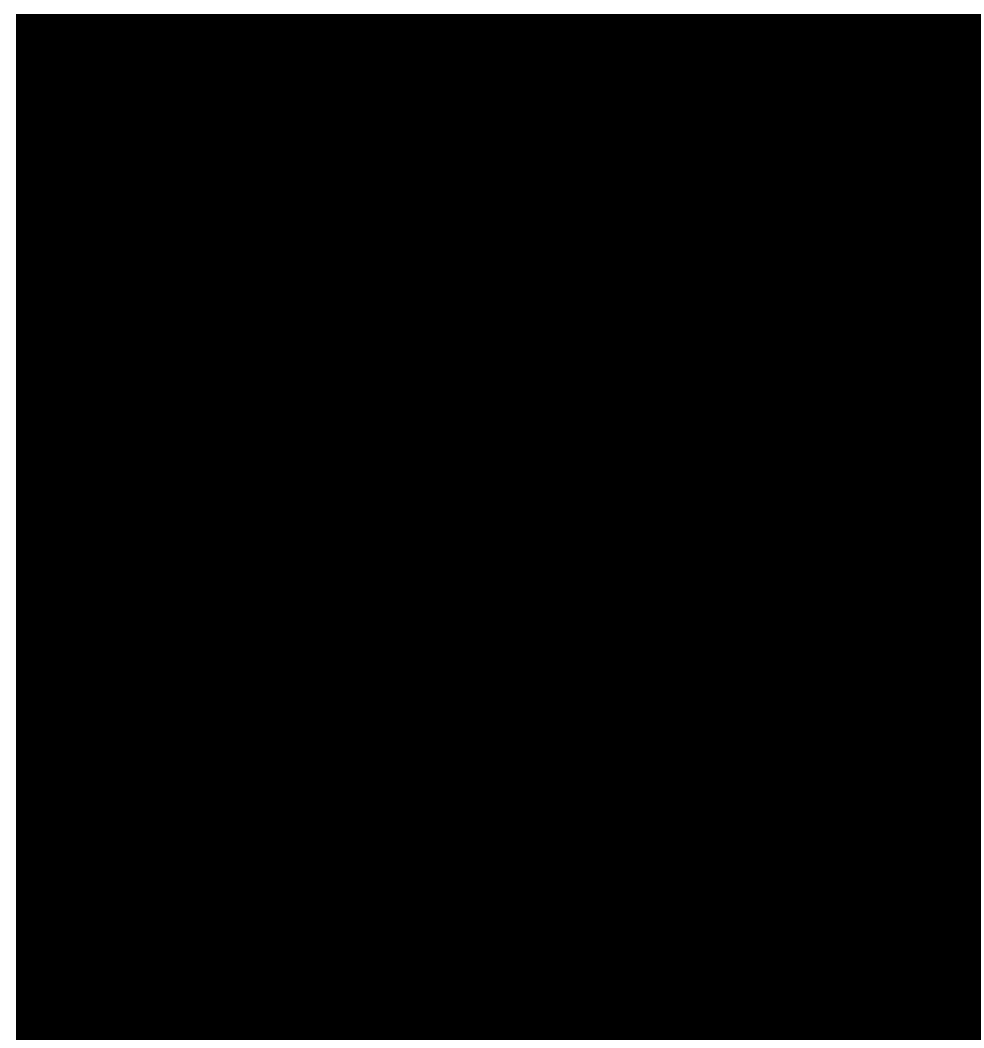

Figure 4.

Sulfide-induced shifts in plasmon resonance on aged nanorods. Nanorods were subjected to one $\mathrm{C} / \mathrm{R}$ cycle at $t=20 \mathrm{~min}$ and aged for several days prior to $\mathrm{Na}_{2} \mathrm{~S}$ addition. Shifts in plasmon resonance (measured immediately after sulfide addition) are as follows: $S: M=1: 2,+0 \mathrm{~nm}$; $\mathrm{S}: \mathrm{M}=1: 1,+20 \mathrm{~nm} ; \mathrm{S}: \mathrm{M}=4: 1,+29 \mathrm{~nm}$. 

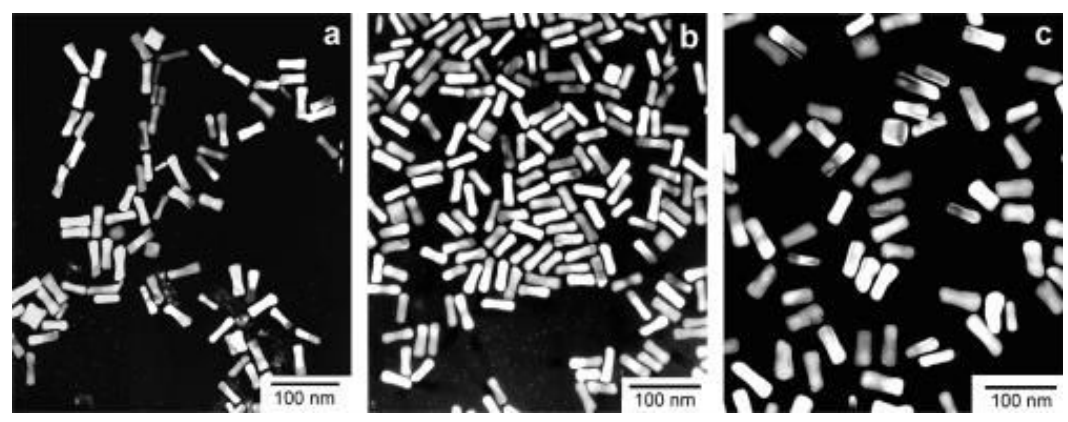

Figure 5.

TEM images (Philips CM-10, $80 \mathrm{kV}$ ) of $\mathrm{Au}$ nanorods after $\mathrm{Na}_{2} \mathrm{~S}$ treatment. Nanorod suspensions were subjected to one $\mathrm{C} / \mathrm{R}$ cycle prior to TEM sample preparation. Reaction times before quenching with $0.2 \mathrm{mM} \mathrm{Na}_{2} \mathrm{~S}$ : (a) $t=15 \mathrm{~min}$; (b) $t=30 \mathrm{~min}$; (c) $t=50 \mathrm{~min}$. 


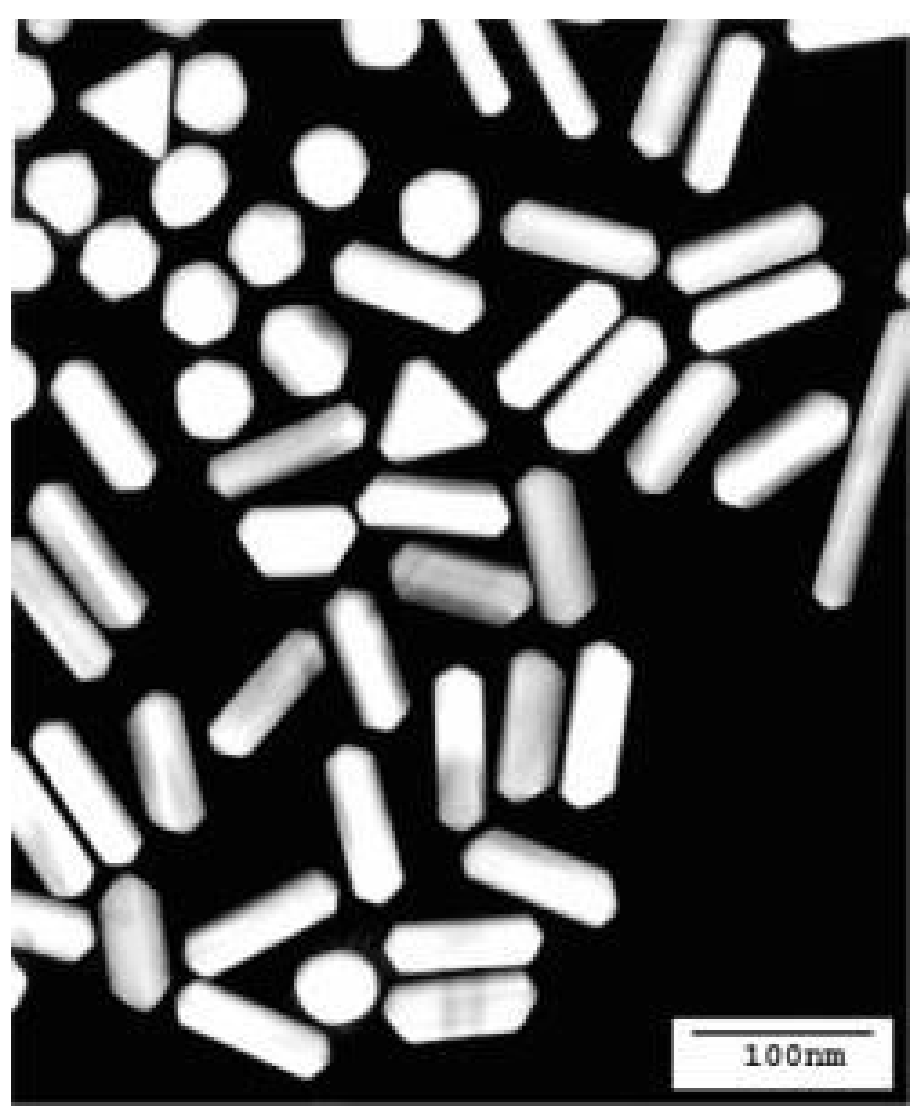

Figure 6.

TEM image (Philips CM-10, $80 \mathrm{kV}$ ) of anisotropic Au nanoparticles prepared in the absence of $\mathrm{AgNO}_{3}$, followed by quenching with $\mathrm{Na}_{2} \mathrm{~S}(t=25 \mathrm{~min})$. Nanorod suspensions were subjected to one $\mathrm{C} / \mathrm{R}$ cycle prior to sample preparation. 


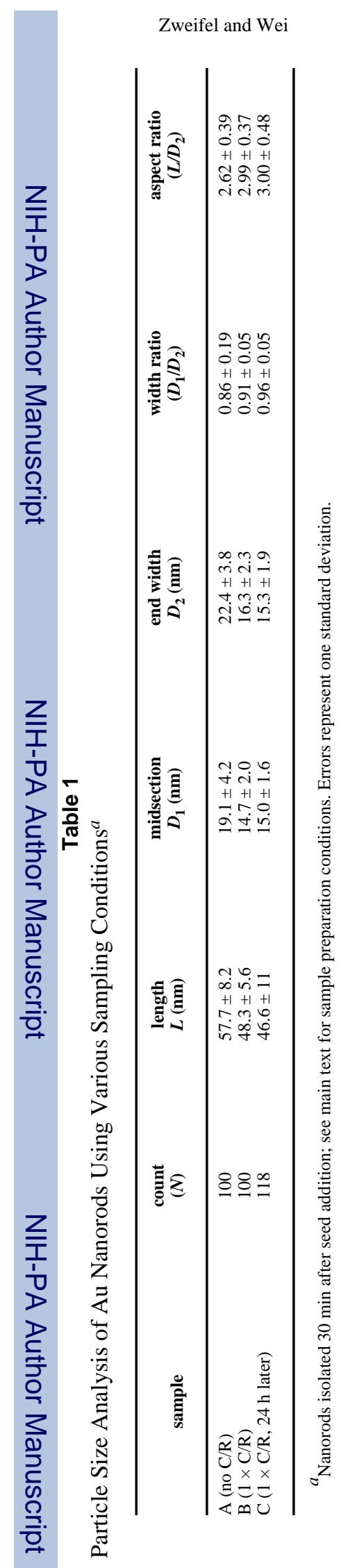

Page 13 


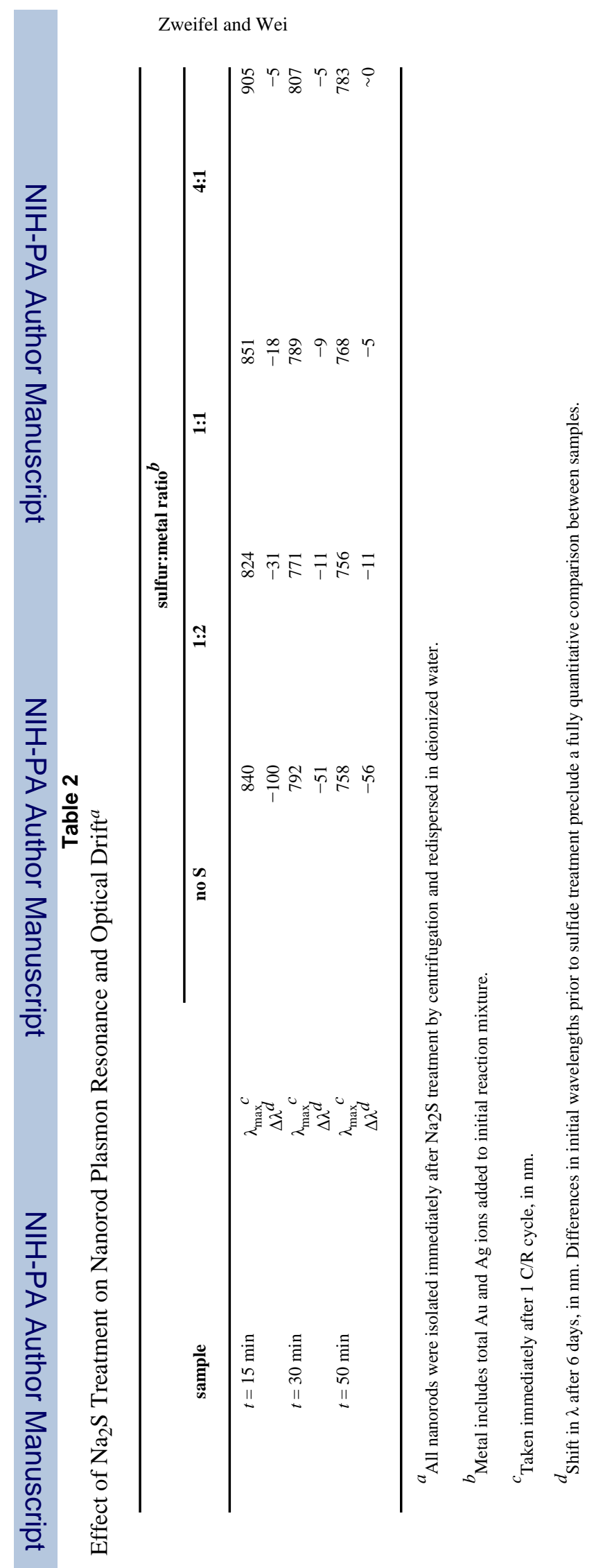

Chem Mater. Author manuscript; available in PMC 2007 April 5. 


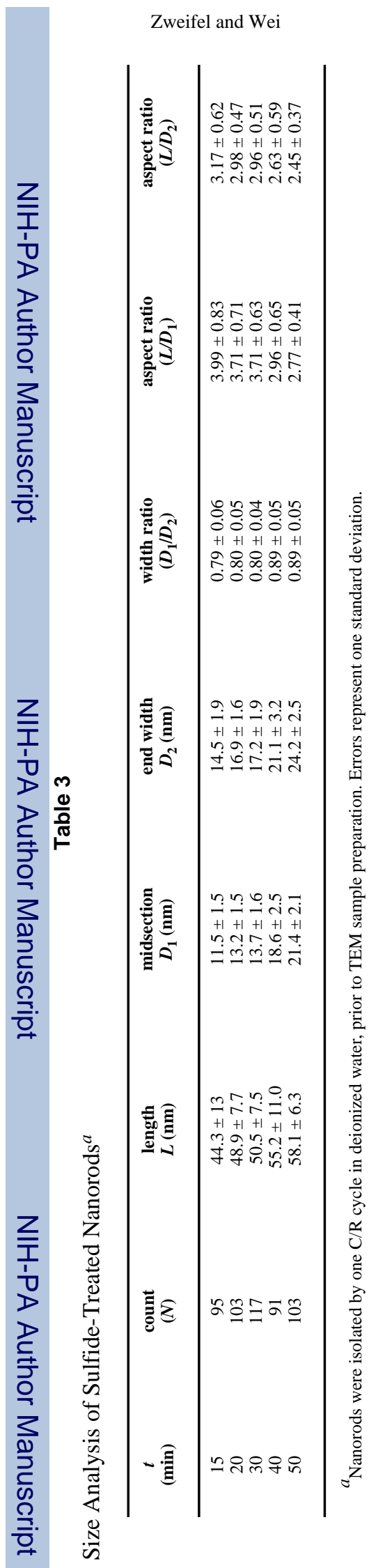

Chem Mater. Author manuscript; available in PMC 2007 April 5. 
Table 4

Longitudinal Plasmon Resonance Analysis of Dumbbell-shaped Nanorods ${ }^{a}$

\begin{tabular}{|c|c|c|c|}
\hline$\underset{(\min )}{t}$ & $\begin{array}{c}\lambda_{\max }, \\
\operatorname{expt}(\mathbf{n m})\end{array}$ & $\begin{array}{c}\lambda_{\max }, \\
\text { calcd using } L / D_{1}^{c}\end{array}$ & $\begin{array}{c}\lambda_{\max }, \\
\text { calcd using } L / D_{2}{ }^{c}\end{array}$ \\
\hline $\begin{array}{l}15 \\
30 \\
50\end{array}$ & $\begin{array}{l}824 \\
771 \\
756\end{array}$ & $\begin{array}{l}806 \\
777 \\
679\end{array}$ & $\begin{array}{l}721 \\
699 \\
646\end{array}$ \\
\hline
\end{tabular}

${ }^{a}$ Nanorods were quenched with $\mathrm{Na}_{2} \mathrm{~S}$ and isolated by one $\mathrm{C} / \mathrm{R}$ cycle in deionized water.

${ }^{b}$ Experimental $\lambda_{\max }$ values taken from Table 2 using $\mathrm{S}: \mathrm{M}=1: 2$.

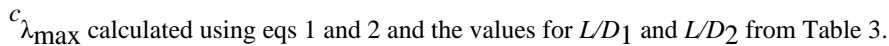

